\title{
Preventive strategy of palliative care during the COVID-19 pandemic period: Strengths, weaknesses, opportunities, and threats analysis
}

\section{Ming-cheng Chung ( $\nabla$ followadan@gmail.com )}

Changhua Christian Medical Foundation Changhua Christian Hospital https://orcid.org/0000-00026892-6112

\section{Pei-Yu Tsai}

Changhua Christian Medical Foundation Changhua Christian Hospital

Jeng-Mei Hsu

Changhua Christian Medical Foundation Changhua Christian Hospital

Chueh-Ko Yang

Changhua Christian Medical Foundation Changhua Christian Hospital

\section{Research article}

Keywords: Preventive strategy, palliative care, COVID-19, pandemic, hospital, home care, SWOT

Posted Date: May 12th, 2020

DOl: https://doi.org/10.21203/rs.3.rs-26603/v1

License: (9) This work is licensed under a Creative Commons Attribution 4.0 International License.

Read Full License 


\section{Abstract \\ Background}

SARS-CoV-2 led to the 2019 coronavirus outbreak (COVID-19) and recently became a pandemic infection. COVID-19's disease mortality rates range from $1 \%$ to above $5 \%$ across many countries and are still rising. Patients receiving palliative care are the most fragile patient group. Palliative care employees treat terminal patients in the hospital, and go into the community to conduct in-home palliative care service. Because palliative care medical staff need to work between the hospital and community every day, they have become a high-risk group for COVID-19 infection.

\section{Methods}

We need a comprehensive care strategy to avoid COVID-19 cross-infection, and protect patients, medical staff, and family members. We divided participants into hospital care and palliative home care to discuss the different strategies. We will use the strengths, weaknesses, opportunities, and threats (SWOT) to analyze preventive strategies.

\section{Results}

We formulated different strategies, mainly by grading and triage, to avoid contact with high-risk latent infections, and used cleaning, disinfection, and personal protective equipment to block possible transmission. Although strict preventive measures had strengths to stop community outbreaks, there are many threats to providing effective palliative care.

\section{Conclusions}

Facing the severe global COVID-19 epidemic, we must have strict strategies to protect our patient and medical staff. But we need to obtain balance between epidemic prevention and palliative care. Preventing the ill patients from feeling lonely or abandoned is also important.

\section{Background}

Severe acute respiratory syndrome coronavirus 2 (SARS-CoV-2), the seventh member of the coronavirus family to infect humans; is an enveloped single-stranded RNA virus. (1) The SARS-CoV-2 caused by the COVID-19 virus and recently became a pandemic infection. In some reports, older patients with comorbid conditions including cardiac disease, pulmonary disease, kidney disease, diabetes, and hypertension were associated with higher mortality rates. (2) According to WHO records, over three million people have been infected with COVID-19 worldwide. Mortality rates range from $1 \%$ to above $5 \%$ across many countries, and are still rising. (2) In Taiwan, our medical system has been on full alert since January 2020, and 
hoped to reduce infection and its progression to severe illness. Patients receiving palliative care are the most fragile patient group. Palliative care employees treat terminal patients in the hospital, and go out into the community for in-home palliative care. This helps patients stay healthy and maintain their quality of life. Because some palliative medical staff move between the hospital and community every day for work, they have become a high-risk group for COVID-19 infection. Therefore, appropriate protection methods are critical to prevent this population from becoming a breach in epidemic infection.

There are three main infection elements: agent, route, and susceptible host. (3) To prevent pathogen exposure, we can screen travel data on hospital entry, and check for fever or pneumonia during initial screens of infected people. Infected individuals can be treated in negative pressure isolation wards, reducing community clusters or the spread of pathogens. However, we cannot avoid those infected during the incubation period, so the next line of defense is to avoid transmission routes, such as droplets and contact transmission, environmental cleaning and disinfection, personal protective equipment worn by medical staff, and proper hand hygiene are all necessary. It is also important for susceptible hosts to avoid cross-infection by regular medical staff and caregivers. We must pay attention to cleaning or disinfection before and after physical contact and when performing procedures.

Therefore, our palliative medical staff developed a complete care strategy to avoid cross-infection of COVID-19 and to protect fragile terminal patients.

\section{Methods}

We divided employees into hospital care and palliative home care groups to discuss the different strategies. The study used SWOT analysis to understand the balance between preventive strategy and palliative and supportive care.

Hospital care

Many people are in and out of the hospital including medical staff, environmental cleaners, patients, caregivers, or family members, etc. Therefore, the first step is simplifying personnel management. At present, with efforts by Taiwan's Center for Disease Control, we can check individuals' immigration records within 30 days through their health insurance card, provide the hospital with accurate travel data and contact history, and avoid asymptomatic infected persons entering and leaving the hospital. 
Table 1

Hospital Measures to Reduce COVID-19 Transmission among palliative care patients

\section{Strategy Content}

Control

Access $\lambda$ Single hospital entrance and exit. An infrared camera monitors the temperature of each employee, patient, or caregiver who enters. If the recorded temperature on the infrared camera exceeds 38 degrees Celsius, the person will be rechecked with a forehead thermometer. If the forehead thermometer is still above 37.5 degrees Celsius, entry is prohibited. And he/she will be referred to the emergency room.

$\lambda$ All entrants, except employees, need to read the immigration information on their health insurance card. If they return from a high-risk area within 14 days, they should not enter the hospital during the incubation period.

$\lambda$ Every entrant is registered and managed by the computer system to prevent crossinfection in the hospital.

Patient and family triage

$\lambda$ Employees can enter with their identification card after their temperature is taken. If the employee has fever or respiratory symptoms, he/she will take sick leave and undergo self-isolation.

$\lambda$ General outpatients can enter the hospital after clearing the fever and travel history restrictions.

$\lambda$ Patients with a fever enter from the emergency room and need to be checked and treated to exclude the possibility of atypical pneumonia.
Medical staff washing hands and protecting

$\lambda$ Alcoholic hand sanitizer is available outside the palliative ward, beside each bed, and on the nursing cart. You must disinfect the front and back of your hands and your wrists with alcohol before and after visiting a patient or performing a treatment.

$\lambda$ Every medical staff wears a new surgical mask every day at the hospital.

$\lambda$ When performing nursing care with secretions and discharge, wear waterproof gowns and goggles.

$\lambda$ At present, the ward's original shifts, team meetings, and activities have been suspended and changed to a small shift mode of 2 to 3 people to reduce the chance of grouping.

Environmental cleaning and disinfection $\lambda$ The elevator should be cleaned with bleach water every hour.

$\lambda$ Daily disinfection of public areas.

$\lambda$ Empty wards should be disinfected with a UV lamp.

$\lambda$ Patient or family time should be reduced in public areas during this period

$\lambda$ Suspended use of the public bathtub

$\lambda$ Beds should be separated by curtains to reduce infection risk 


\begin{tabular}{|c|c|}
\hline Strategy & Content \\
\hline \multirow{3}{*}{$\begin{array}{l}\text { Regular } \\
\text { caregiver or } \\
\text { family } \\
\text { member }\end{array}$} & $\begin{array}{l}\lambda \text { Each caregiver or family member will check the health insurance card to confirm } \\
\text { travel and contact history, and the nursing station will register the list uniformly. }\end{array}$ \\
\hline & $\begin{array}{l}\lambda \text { In general wards, there can be only one family member or caregiver care one } \\
\text { patient. }\end{array}$ \\
\hline & $\begin{array}{l}\lambda \text { If you cannot visit or are isolated at home, you can use video chat to contact the } \\
\text { patient }\end{array}$ \\
\hline \multirow{4}{*}{$\begin{array}{l}\text { Patient care } \\
\text { and } \\
\text { monitoring }\end{array}$} & $\lambda$ Monitor the body temperature and vital signs of patients every eight hours \\
\hline & $\begin{array}{l}\lambda \text { If patients with a fever are screened for its cause, the chest X-ray will be checked } \\
\text { to rule out pneumonia. }\end{array}$ \\
\hline & $\lambda$ If fever source is confirmed, we will provide the appropriate treatment. \\
\hline & $\begin{array}{l}\lambda \text { If those who are highly suspected of a COVID-19 infection, have a history of } \\
\text { exposure, or have pneumonia that is ineffective against antibiotics, we will consult } \\
\text { the Infectious Diseases Department. The patient will first transfer to a separate } \\
\text { ward or negative pressure isolation ward to see if further testing is needed. }\end{array}$ \\
\hline $\begin{array}{l}\text { Ambient } \\
\text { temperature } \\
\text { and humidity }\end{array}$ & $\begin{array}{l}\lambda \text { The COVID-19 epidemic started in December } 2019 \text {, so it is speculated the virus } \\
\text { can survive longer in colder environments. (4) Therefore, the central air conditioner } \\
\text { of this hospital is set at } 25 \text { degrees } C \text { and the relative humidity is about } 50 \sim 60 \% \text { to } \\
\text { reduce the virus survival time. }\end{array}$ \\
\hline
\end{tabular}

\section{Palliative home care}

Because palliative medical staff need to leave the hospital and go out into the community for home care, we need fewer access and contact history controls. Our medical staff are exposed to a relatively high risk of infection. Therefore, according to the policy of Center for Disease Control and the standard operating procedures of our hospital, we need strategies to protect our patients and paramedics from crossinfection. 
Table 2

Palliative Home Care COVID-19 Protection Measures

\section{Strategy Content}

Before home $\quad \lambda$ Before visiting a new patient, the home-care nurse will contact the caregiver by care phone to confirm patient's condition. The nurse will ask if there is a family member who has returned from abroad within the last 14 days. If any isolating family members at home or patient has a fever, we will stop arranging visits, and the patients will be advised to return to emergency room department for screening.

$\lambda$ If there is no fever, contact history, or it is an old case, we will arrange palliative home care service.

$\lambda$ We recommend returning to the hospital for repeated incidence of fever, ineffective antibiotics treatment, or suspected pneumonia.

$\lambda$ Before our medical staff enters the home, we have to monitor our body temperature and upper respiratory symptoms daily. If there are any abnormalities, the nurse must cancel or postpone home care time to avoid transmitting the virus from the hospital to homes.

During home care $\lambda$ Medical staff need to wear glasses, and those without myopia need to wear goggles.

$\lambda$ Before and after touching the patient or performing a technique, the front and back of the hand and the wrist must be disinfected with alcohol sanitizer.

$\lambda$ Wear surgical masks and waterproof gowns. When leaving a patients' place, waterproof gowns must be taken off and wrapped in red plastic bags, and brought back to the hospital for high-pressure, high-temperature disinfection and cleaning.

After home $\quad \lambda$ The nurse will call each week to confirm the patient's temperature change and care upper respiratory symptoms.

Environmental cleaning and disinfection $\lambda$ We recommend patients' rooms and public areas be cleaned and disinfected daily with diluted bleach (500 ppm, about $0.05 \%)$.

$\lambda$ Ask family members or caregivers to clean the front and back of the hands and wrists with alcohol sanitizer or soap before and after touching the patient.

SWOT analysis

A SWOT analysis was applied to understand the strengths, weaknesses, opportunities and threats of preventive strategies in palliative and end-of-life care. A SWOT approach provides an overall discussion of these issues. (5) We recorded the debate of preventive strategies according to the conference of our palliative team members.

\section{Results}

A summary of the SWOT analysis is presented in Table 3. 
Table 3

Strengths, weaknesses, opportunities, and threats of infection preventive strategies in palliative and supportive care

\section{Strengths}

$\lambda$ Control access reduces the entry of people who are high risk

$\lambda$ Block possible transmission routes, and only one person, a family member or caregiver, can be at the bedside

$\lambda$ Increase hand washings and personal protective equipment use among medical staff

$\lambda$ Reduce community grouping and infection agent spread

$\lambda$ Protect medical staff and vulnerable patients

\section{Opportunities}

$\lambda$ Increased personal and environmental cleanliness: reduces patient infection risk, such as a declined influenza infection rate

$\lambda$ Increased video visits, the hospital provides a wireless connection environment, so the distant family member can communicate through the network

\section{Weakness}

$\lambda$ As long as the caregiver has a fever or upper respiratory tract symptoms, they cannot accompany patients

$\lambda$ Patients and family members spend less time together, and all family members cannot accompany them

$\lambda$ Medical staff will have increased dressing time and psychological pressure using personal protective equipment when contacting patients or family members

\section{Threats}

$\lambda$ Strict medical care is much distant and less humanized

$\lambda$ The patient's psychological and spiritual needs cannot be satisfied because of reduced outings, hospital activities or space use

$\lambda$ It is harder for patients to die a good death.

$\lambda$ Increased family complaints and reduced companionship time

$\lambda$ Reduced intimacy and companionship between patients and their families

At present, there are more than 430 people diagnosed with COVID-19 in Taiwan. Most cases were imported from abroad. Although strict home quarantine measures have been implemented for returnees, we must still be careful of community outbreaks. Hospital entry and exit is very complicated. Therefore, there are strict control measures. So far, there has been no cross-infection between home and hospital in Taiwan. Our palliative unit also needs to take care of patients, caregivers, and medical staff in the hospital. In addition, our patients receive palliative home care after being discharged, which creates direct exposure to many unknown risks. Our standard operating procedure of care is shared for everyone. We hope that all palliative patients and medical staff can be protected from the threat of COVID-19 infection. We need to protect ourselves and give our patients more comfortable care.

\section{Conclusions:}

We serve about 500 palliative inpatients every year and conduct nearly 3,000 home visits a year. Facing the severe global epidemic of COVID-19, we must have strategies to protect our patients and medical 
staff. The pandemic will interrupt ward and home care service. Medical staff will need to be quarantined or even treated. This will risk the health of fragile and terminal patients and prevent them from receiving good palliative care and maintaining their health. We took rigorous preventive measures, and reduced important family support and care in the palliative care. It is very important to prevent patients from feeling lonely or abandoned. This is what we need to obtain balance between epidemic prevention and palliative care.

We have formulated different strategies for palliative ward and home care to prevent the spread of COVID-19, mainly by grading and triage. These measures avoid contacting with high-risk latent infections, and using cleaning, disinfection, and personal protective equipment to block possible transmission routes, ultimately protecting our palliative patients that are more susceptible infection. We need to provide patients with peace of mind by explaining the pros and cons of these protective measures.

\section{Abbreviations}

\section{SARS-CoV-2}

Severe acute respiratory syndrome coronavirus 2

COVID-19

Corona Virus Disease 2019

WHO

World Health Organization

SWOT

strengths, weaknesses, opportunities, and threats analysis

UV

ultraviolet

\section{Declarations}

\section{Availability of data and materials}

All data reported are included in this published article. The preventive strategies and analysis during the current study is available from the corresponding author on reasonable request.

\section{Acknowledgements}

The authors would like to thank all members of department of hospice and palliative medicine in Changhua Christian hospital, for caring our hospice patients and sacrificing themselves.

\section{Funding}

The author(s) have no receipt of the financial support for the report, authorship, and/or publication of this article 


\section{Author information}

\section{Affiliations}

Department of hospice and palliative medicine, Changhua Christian hospital.

No. 135, Nanxiao St., Changhua City, Changhua County 500, Taiwan (R.O.C.)

Ming-Cheng Chung ${ }^{1}$, Pei-Yu Tsai ${ }^{1}$, Jeng-Mei Hsu

Biosafety committee, Changhua Christian Hospital.

No. 135, Nanxiao St., Changhua City, Changhua County 500, Taiwan (R.O.C.)

Chueh-Ko Yang

\section{Contributions}

Ming-Cheng Chung drafted the manuscript. Pei-Yu Tsai led the SWOT analysis in our palliative conference for preventive strategies formulation. Jeng-Mei Hsu help to record the conference. Chueh-Ko Yang added some hospital policy into the palliative preventive strategies. All authors revised the manuscript critically and read and approved the final manuscript.

\section{Corresponding author}

Correspondence to Ming-Cheng Chung

\section{Ethics declarations}

\section{Ethics approval and consent to participate}

In this analysis, no medical research involving patients or family caregivers was performed. Therefore, the approval of local ethics committee was not required. All participants and their discussion data would be anonymously stored and processed.

\section{Consent for publication}

Not applicable. The manuscript does not contain data from any individual person.

\section{Competing interests}

The authors declare that they have no competing interests.

\section{References}


1. Zhu N, Zhang D, Wang W, Li X, Yang B, Song J, et al. A Novel Coronavirus from Patients with Pneumonia in China, 2019. N Engl J Med. 2020;382(8):727-33.

2. Patel AB, Verma A. COVID-19 and Angiotensin-Converting Enzyme Inhibitors and Angiotensin Receptor Blockers: What Is the Evidence? JAMA. 2020.

3. Organization WH. Infections and infectious diseases: a manual for nurses and midwives in the WHO European Region. World Health Organization. 2001;No. EUR/01/5019329.

4. Kampf G, Todt D, Pfaender S, Steinmann E. Persistence of coronaviruses on inanimate surfaces and their inactivation with biocidal agents. J Hosp Infect. 2020;104(3):246-51.

5. Gürel E, Tat MJJoISR. SWOT analysis: a theoretical review. 2017;10(51). 